\title{
Evaluation of the cardiotoxicity and resuscitation of rats of a newly developed mixture of a QX-3/4 analog and levobupivacaine
}

This article was published in the following Dove Press journal:

Journal of Pain Research

27 March 2017

Number of times this article has been viewed

\author{
Qi Wang' \\ Qinqin Yin' \\ Jun Yang ${ }^{2}$ \\ Bowen $\mathrm{Ke}^{2}$ \\ Linghui Yang ${ }^{2}$ \\ Jin Liu',2 \\ Wensheng Zhang ${ }^{1,2}$ \\ 'Department of Anesthesiology, \\ ${ }^{2}$ Laboratory of Anesthesia and \\ Critical Care Medicine, Translational \\ Neuroscience Centre, West China \\ Hospital, Sichuan University, Chengdu, \\ Sichuan, People's Republic of China
}

Correspondence: Wensheng Zhang

Department of Anesthesiology, West

China Hospital, Sichuan University,

Chengdu 6I004I, Sichuan, People's

Republic of China

Tel +862885164040

Email zhang_ws@scu.edu.cn
Objective: This study was designed to evaluate the cardiotoxicity of a QX-314 analog (QX-OH) and a mixture of QX-OH and levobupivacaine (LL-1) and to compare the ability to resuscitate rats after asystole induced by levobupivacaine (Levo-BUP), QX-314, QX-OH, and LL-1.

Methods: First, we used the "up-and-down" method to determine median dose resulting in appearance of cardiotoxicity $\left(\mathrm{CD}_{50 \mathrm{C}}\right)$ and asystole $\left(\mathrm{CD}_{50 \mathrm{~A}}\right)$ of Levo-BUP, QX-314, QX-OH, and LL-1 in rats. Safety index (SI; ratio of $\mathrm{CD}_{50 \mathrm{C}}$ compared with 2-fold median effective dose needed to produce sensory blockade) of the 4 drugs was calculated. Isobolograms were used for drug interaction analysis. Second, rats received 1.2-fold $\mathrm{CD}_{50 \mathrm{~A}}$ in the 4 groups. When asystole occurred, standard cardiopulmonary resuscitation was started and continued for $30 \mathrm{~min}$ or until return of spontaneous circulation (ROSC) with native rate-pressure product $\geq 30 \%$ baseline for $5 \mathrm{~min}$.

Results: Ranking of $\mathrm{CD}_{50 \mathrm{C}}$ was Levo-BUP $<\mathrm{QX}-314 \approx \mathrm{QX}-\mathrm{OH}$. Ranking of $\mathrm{CD}_{50 \mathrm{~A}}$ was LevoBUP $<$ QX-314 < QX-OH. However, the SI of Levo-BUP was significantly higher than that of QX-314 (10.60 vs. 1.20) or QX-OH (10.60 vs. 1.44). The SI of LL-1 was similar to that of Levo-BUP. Nonsynergistic interaction was observed for cardiac effects between QX-OH and Levo-BUP. ROSC was attained initially by 8 of 8 rats in the Levo-BUP group, 3 of 8 in the QX-314 group, 6 of 8 in the QX-OH group, and 8 of 8 in the LL-1 group. Sustained recovery was achieved in the Levo-BUP group but not in the other groups.

Conclusion: Levo-BUP and LL-1 are safer than QX-314 or QX-OH. Cardiac effects between QX-OH and Levo-BUP were nonsynergistic. Initial successful resuscitation could be achieved in the QX-OH- and LL-1-induced asystole, but advanced life support might be needed.

Keywords: asystole, cardiotoxicity, levobupivacaine, QX-314, QX-OH

\section{Introduction}

QX-314 (N-[2,6-dimethylphenyl carbamoylmethyl] triethylammonium bromide), a quaternary of lidocaine (LIDO), is difficult to permeate through cell membranes because it has a permanent positive charge (Figure 1A). Nevertheless, some studies have shown that QX-314 can produce long-lasting local anesthesia with a slow onset. ${ }^{1,2}$ Therefore, the key is to improve the onset time of QX-314. The transient receptor potential (TRP) was found to be the primary pathway of QX-314 to elicit local anesthesia. ${ }^{3}$ An agonist of TRP, capsaicin, can promote the effects of QX-314. ${ }^{4}$ However, capsaicin is limited for painful irritation and toxicity in local tissue. ${ }^{5}$

QX-314 produces long-lasting nerve blockade after a delayed onset when administered extracellularly as a peripheral local anesthetic. To improve its penetrability and accelerate the drug entry into cells, a derivative of QX-314, QX-OH (2-(2,6-dimethylphenylamino)- $N, N$-diethyl- $N$-(2-hydroxyethyl)-2-oxoethanaminium 
A

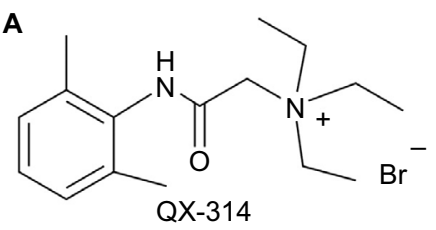

B

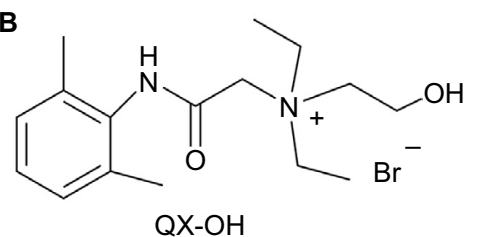

Figure I Molecular structure of (A) QX-314 and (B) QX-OH.

Notes: QX-OH is different from QX-3/4 for the presence of an additional hydroxyl group $(-\mathrm{OH})$ attached to the amine moiety.

bromide; molecular weight, 359.3; Figure 1B), has been synthesized independently. Previously, we showed in animal models that QX-OH alone can (like QX-314) not only produce long-lasting local anesthesia but also moderate toxicity in local tissue compared with the same concentration of QX-314. However, the onset time of QX-OH alone was still not improved significantly.

Bupivacaine (BUP), a local anesthetic with fast onset, can activate TRP channels in the same way as capsaicin, inducing cellular entry of QX-314. ${ }^{6}$ Therefore, we expect co-application of QX-OH and BUP to induce a prolonged blockade with rapid onset. Upon consideration of the severe system toxicity of BUP, we choose the single levorotatory isomer of BUP, levobupivacaine (Levo-BUP), which is safer than BUP. Therefore, we conducted research on QX-OH, as well as a mixture of QX-OH and Levo-BUP, to meet the need for long-acting local anesthetics.

Previously, we found that $35 \mathrm{mM} \mathrm{QX}-\mathrm{OH}$ in combination with 10 levobupivacaine, which we referred to as "LL-1", produced long-lasting sciatic nerve blockade in rats, which can be as long as $12 \mathrm{~h}$, almost 3 times longer than $0.75 \%$ (23 mM) BUP alone, with minimal to mild local histological changes that were similar to those seen with $0.75 \%$ BUP. Moreover, the onset time of LL-1 was acceptable ( $<10 \mathrm{~min}$ ). However, cardiotoxicity may be induced by QX-OH or LL-1 (just as with other local anesthetics). Severe cardiotoxicity after administration of local anesthetics always is lethal. ${ }^{7}$ Therefore, it is crucial to evaluate the cardiotoxicity of QX-OH and LL-1.

The potency of cardiotoxicity is closely related to the lipid solubility of local anesthetics. The lipid solubility of QX-OH is less than that of QX-314 due to the changes in chemical structure. Therefore, we hypothesized that the cardiotoxicity of QX-OH was less than that of QX-314 due to the improvements in physicochemical properties. In addition, whether QX-OH combined with Levo-BUP can increase cardiotoxicity is not known. Therefore, we compared the cardiotoxicity of Levo-BUP, QX-314, and QX-OH in anesthetized rats and assessed the interaction between Levo-BUP and QX-OH. We also evaluated the ability to resuscitate rats upon asystole after administration of these local anesthetics.

\section{Methods}

\section{Animals}

Male and female Sprague Dawley rats (230-350 g) were provided by the Experimental Animal Centre of Sichuan Province. Rats were housed in cages with free access to food and water and were maintained on a 12-h light-dark cycle. Rats had a $\geq 3$-day acclimatization period in the animal facility before initiation of experiments. The study was approved by the Committee of Animal Care of West China Hospital, Sichuan University, People's Republic of China (approval number: 2015014A). The study was carried out in accordance with the Guide for the Care and Use of Laboratory Animals (US National Institutes of Health; NIH publication number 80-23 [revised 1996]).

\section{Drugs}

QX-314 was purchased from Sigma-Aldrich Canada Ltd. (Oakville, Ontario, Canada). QX-OH was synthesized by our research team. Levo-BUP was obtained from Jiangsu Hengrui Medicine Co., Ltd (Jiangsu, People's Republic of China). Levo-BUP (10 $\mathrm{mM}$ ) was prepared by dilution with physiologic saline. QX-314 (35 mM) and QX-OH (35 mM) were dissolved in physiologic saline. LL-1 was prepared by mixing of QX-OH and Levo-BUP at a proportion of 35:10 mM.

\section{Experiment I: Determination of median dose resulting in appearance of cardiotoxicity $\left(C D_{50 C}\right)$ and safety index (SI) of Levo-BUP, QX-3।4, $\mathrm{QX}-\mathrm{OH}$, and LL-I}

$\mathrm{CD}_{50 \mathrm{C}}$ of Levo-BUP, QX-314, QX-OH, and LL-1 was measured using the "up-and-down" method. ${ }^{8}$ This method, known as the "staircase bioassay", is a procedure that has been proved to be effective in reducing the number of animals needed to determine a median dose. This method is appropriate for a variety of experimental end points, including toxicity studies. In the course of the experiment, if a positive result is observed, the subsequent dose of the study drug will be decreased by the dosing increment. If a negative result occurs, the dose will be increased. This "up-and-down" procedure is repeated until 5 crossovers (negative to positive or positive to negative effect) are obtained. 
Rats were anesthetized in a bell jar with $2 \%$ isoflurane in $100 \%$ oxygen. Upon no aversive response to a tail-clamping stimulus, rats were then placed on a board under a warming lamp to maintain body temperature at $37^{\circ} \mathrm{C}-38^{\circ} \mathrm{C}$. Spontaneous breathing was maintained with $1 \%$ isoflurane. Three-lead electrocardiogram (ECG) was initiated by subcutaneous placement of needle electrodes at the left upper limb, right upper limb, and left lower limb. ${ }^{2}$ Electrocardiographic data were acquired and displayed using a portable biological function experimental system (BL-420F; Taimeng Science and Technology Ltd., Chengdu, Sichuan, People's Republic of China) equipped with alternating current and $50 \mathrm{~Hz}$ filters and connected to a computer running the recording software. Then, venipuncture was undertaken on the distal third of the tail with a $22 \mathrm{G}$ intravenous cannula (Terumo Medical Corporation, Tokyo, Japan).

After a stable period of $5 \mathrm{~min}$, the study drugs were injected. After injection, we observed electrocardiographic evidence of the toxic effects on cardiac automaticity, conductivity, and rhythmicity for $15 \mathrm{~min}$. A "positive" result was defined as a change in the following end points: a decrease in baseline heart rate $\geq 30 \%$, any evidence of atrioventricular conduction blockade, widening of the QRS complex, or other arrhythmias. ${ }^{9}$ For Levo-BUP, the starting dose was $1 \mathrm{mg} / \mathrm{kg}$ with a $\log$ dosing increment of 0.181 . For QX-314, the starting dose was $5 \mathrm{mg} / \mathrm{kg}$ with a $\log$ dosing increment of 0.095 . For QX-OH, the starting dose was $5 \mathrm{mg} / \mathrm{kg}$ with a log dosing increment of 0.106 . For LL-1, the starting dose (QX-OH) was $4 \mathrm{mg} / \mathrm{kg}$ with a log dosing increment of 0.095 .

To evaluate SI, we need to consider the tested drugs potency for local anesthesia. In rat sciatic nerve block model, we used a hot plate experiment to determine the median effective dose required to produce sensory blockade $\left(\mathrm{ED}_{50}\right)$. Briefly, rats were anesthetized with $2 \%$ isoflurane for $\sim 1$ min. Rats were placed a left lateral position. An injection (23 G, 1 inch hypodermic needle) was vertically inserted in the center of the line linking trochanter major and tuber ischiadicum of left leg. The tested drug was injected when bone was contacted. Sensory block was measured in the same anatomic area using a modified hot plate (model RB-200 hot plate analgesia meter; Chengdu Technology \& Market Co., Ltd.) at a temperature of $56^{\circ} \mathrm{C}$. A "positive" result was defined as the time that the animal left its paw was beyond $6 \mathrm{~s} .{ }^{4}$ All rats were euthanized with isoflurane after each experiment.

\section{Experiment 2: Determination of median dose resulting in asystole $\left(C D_{50 \mathrm{~A}}\right)$ of Levo-BUP, QX-3 I4, QX-OH, and LL-I}

The method employed to measure the $\mathrm{CD}_{50 \mathrm{~A}}$ of LevoBUP, QX-314, QX-OH, and LL-1 was the same as that for experiment 1 except for 3 differences. First, the trachea was cannulated via tracheostomy, and then lungs were ventilated using a piston ventilator with a tidal volume of $10-12 \mathrm{~mL} /$ $\mathrm{kg}$ at $50-55$ breaths/min to maintain the normal pressure of end-tidal carbon dioxide pressure. Second, a positive result was defined as a lack of a recognizable beat on ECG for 1 min after appearance of the last systole or occurrence of ventricular fibrillation. ${ }^{10,11}$ Third, based on our preliminary experiment, we needed to observe $60 \mathrm{~min}$. The starting dose was $6 \mathrm{mg} / \mathrm{kg}$ with a log dosing increment of 0.119 for LevoBUP. For QX-314, the starting dose was $30 \mathrm{mg} / \mathrm{kg}$ with a $\log$ dosing increment of 0.084 . For QX-OH, the starting dose was $40 \mathrm{mg} / \mathrm{kg}$ with a log dosing increment of 0.059 . For LL-1, the starting dose (QX-OH) was $20 \mathrm{mg} / \mathrm{kg}$ with a log dosing increment of 0.083 .

\section{Experiment 3: Resuscitation after induction of asystole with Levo-BUP, QX-3 |4, QX-OH, or LL-I}

Rats of either sex were anesthetized with $2 \%$ isoflurane. The trachea was cannulated via tracheostomy, and lungs were ventilated mechanically as described in experiment 2 . ECG was recorded, and body temperature was maintained at $37^{\circ} \mathrm{C}-38^{\circ} \mathrm{C}$ using a heating lamp. The left femoral artery was cannulated for blood sampling and monitoring of arterial pressure. Through the left femoral vein, a cannula was placed into the vena cava for injection of epinephrine. The tail vein was cannulated for administration of local anesthetics.

After surgical preparation, rats were allowed to stabilize for $5 \mathrm{~min}$ in an atmosphere of 1\% isoflurane and 100\% oxygen. Arterial blood gas was measured to confirm a $\mathrm{pH}$ between 7.30 and 7.45 and a serum lactate $<2.0 \mathrm{mmol} / \mathrm{L}$ before administration of local anesthetics. According to our preliminary experiment, rats were assigned randomly to receive 1.2-fold $\mathrm{CD}_{50 \mathrm{~A}}$ in the 4 groups - a dose that reliably produces asystole from which hemodynamic recovery will occur with cardiopulmonary resuscitation. Resuscitation was started if asystole occurred. If asystole did not occur, the rat was excluded. Finally, 8 rats were needed in each group.

Isoflurane was discontinued if asystole occurred. External cardiac compressions were applied immediately at a rate of $\sim 300$ beats/min to produce a systolic arterial pressure $>40 \mathrm{mmHg}$ until spontaneous rhythm returned and were interrupted for $5 \mathrm{~s}$ every $1 \mathrm{~min}$ to evaluate circulatory function. Simultaneously, epinephrine $10 \mu \mathrm{g} / \mathrm{kg}$ was administered at 1-min intervals until return of spontaneous circulation (ROSC), which was defined as rate-pressure 
product (RPP; systolic pressure $\times$ heart rate) $\geq 30 \%$ of the baseline value for $5 \mathrm{~min}$. Anesthesia was restarted after ROSC. Resuscitation was stopped if ROSC was not observed after $30 \mathrm{~min}$. ECG and arterial pressure were monitored for $60 \mathrm{~min}$ continuously. Samples of arterial blood $(0.3 \mathrm{~mL})$ were drawn in heparinized syringes before administration of local anesthetics (baseline) as well as $15,30,45$, and $60 \mathrm{~min}$ after ROSC for the measurement of arterial blood gases.

The following data were also collected in all 4 groups: time from initiation of injection of local anesthetics to asystole $\left(\mathrm{T}_{\text {asystole }}\right)$; time from the start of asystole to ROSC $\left(\mathrm{T}_{\text {recovery }}\right)$; dose of epinephrine required for successful resuscitation; duration of arrhythmia (\%) after ROSC (if abnormal ECG was accompanied by abnormal arterial pulsation for 1 screen, this period was included in the duration of arrhythmia).

\section{Statistical analysis}

Data were analyzed using SPSS v19.0 (IBM Corporation, Armonk, NY, USA). Differences in the $\mathrm{CD}_{50 \mathrm{C}}$ and $\mathrm{CD}_{50 \mathrm{~A}}$ among groups were considered significant if $95 \%$ confidence intervals did not overlap. ${ }^{12}$ The SI was calculated by using the equation:

$$
\frac{\mathrm{CD}_{50 \mathrm{C}}}{2 \mathrm{ED}_{50}}=\mathrm{SI}
$$

Isobolographic analysis was used to evaluate the interaction between Levo-BUP and QX-OH. ${ }^{13,14}$ The interaction index $(\gamma)$ indicates the changed potency of a combination and is defined using the equation:

$$
\frac{\mathrm{a}}{\mathrm{A}}+\frac{\mathrm{b}}{\mathrm{B}}=\gamma
$$

where $\mathrm{A}$ and $\mathrm{B}$ are the doses of drug A (alone) and drug B (alone), respectively, that give the specified effect and $(a, b)$ is the combination dose that produces the same effect. If $\gamma=1$, the interaction is additive; if $\gamma<1$, it is synergistic; if $\gamma>1$, it is antagonistic.
For experiment 3, sample size calculation was based on the results of previous experiments comparing survival rate at 10 min after ROSC among various groups. In a CochranArmitage test for trend in proportions, sample sizes of 8,8 , 8 , and 8 are obtained from 4 groups with equally spaced $\mathrm{X}$ values and proportions equal to $0.80,0.80,0.40$, and 0.20 (survival rate obtained from previous studies), respectively. The total sample of 32 subjects achieves $80 \%$ power to detect a linear trend using a 2 -sided $Z$ test with continuity correction and a significance level of 0.05 .

Data are the mean \pm standard deviation (SD). Baseline parameters of cardiac function, weight, $T_{\text {asystole }}, T_{\text {recovery }}$, dose of epinephrine required for successful resuscitation, and duration of arrhythmia (\%) after ROSC among groups were analyzed by 1-way analysis of variance and Scheffe post hoc tests. Variables in arterial blood gas among groups after ROSC were analyzed across time by analysis of variance with repeated-measures and Scheffe post hoc tests. Survival rates were analyzed by Fisher's exact test. $P<0.05$ was considered significant.

\section{Results}

\section{The $C D_{50 C}$ of Levo-BUP, QX-3।4, $\mathrm{QX}-\mathrm{OH}$, and LL-I}

The baseline heart rate of rats was $367 \pm 28,367 \pm 28,355 \pm$ 46 , and $358 \pm 38$ beats/min in Levo-BUP, QX-314, QX-OH, and LL-1 groups, respectively $(P>0.05)$. In all groups, a decrease in heart rate and widening of the QRS complex after administration of study drugs was observed. All rats that tested positive for cardiotoxicity still survived after experiment. The $\mathrm{CD}_{50 \mathrm{C}}$ for different groups is summarized in Table 1. The $\mathrm{CD}_{50 \mathrm{C}}$ was significantly lower for Levo-BUP than for QX-314 or QX-OH. No significant difference was seen between QX-314 and QX-OH groups.

\section{The $\mathrm{CD}_{50 \mathrm{~A}}$ of Levo-BUP, QX-3।4, $\mathrm{QX}-\mathrm{OH}$, and LL-I}

Baseline heart rates were similar among groups $(P>0.05)$. Rats with a positive result developed asystole quickly after

\begin{tabular}{|c|c|c|c|c|}
\hline Groups & $E D_{50}(\mathrm{mg} / \mathrm{kg})$ & $\mathrm{CD}_{50 \mathrm{C}}$ and $95 \% \mathrm{Cl}$ (range) (mg/kg) & SI (range) & $\mathrm{CD}_{50 \mathrm{~A}}$ and $95 \% \mathrm{Cl}$ (range) (mg/kg) \\
\hline I Levo-BUP & 0.14 & $2.97(2.30-3.85)$ & $10.60(8.21-13.75)$ & $19.31(16.52-22.57)$ \\
\hline II QX-3|4 & 4.17 & $10.00(9.13-10.94)$ & $1.20(1.09-1.31)$ & $62.37(58.16-66.89)$ \\
\hline III QX-OH & 3.60 & $10.40(9.34-11.57)$ & $1.44(1.30-1.61)$ & $78.17(74.46-82.08)$ \\
\hline IV Levo-BUP & 0.10 & $2.17(1.92-2.45)$ & $10.85(9.60-12.25)$ & $11.87(9.91-14.20)$ \\
\hline QX-OH & 0.43 & $8.39(7.43-9.47)$ & $9.76(8.64-11.01)$ & $45.95(38.35-54.98)$ \\
\hline
\end{tabular}

Table I $C D_{50 C}, C D_{50 A}$, and $\mathrm{SI}$ of all 4 groups

Notes: $C D_{50 C}$, median dose resulting in appearance of cardiotoxicity; $C D_{50 A}$, median dose resulting in asystole; ED ${ }_{50}$, median effective dose required to produce sensory block; Group IV, LL-I (mixture of QX-OH and Levo-BUP); SI, safety index (ratio of drug doses required to produce cardiotoxicity compared with 2-fold ED ${ }_{50}$ ). Abbreviations: $\mathrm{Cl}$, confidence interval; Levo-BUP, levobupivacaine. 
administration of Levo-BUP or LL-1. However, all rats that tested positive developed ventricular fibrillation a few minutes after administration of QX-314 or QX-OH. The $\mathrm{CD}_{50 \mathrm{~A}}$ for Levo-BUP was significantly lower than that for QX-314 or QX-OH. However, the $\mathrm{CD}_{50 \mathrm{~A}}$ of QX-OH was significantly higher than that of QX-314 (Table 1).

\section{The SI of LL-I was similar to Levo-BUP}

The $\mathrm{ED}_{50}$ was $0.10,4.17$, and $3.60 \mathrm{mg} / \mathrm{kg}$ in Levo-BUP, QX-314, and QX-OH groups. For LL-1, the $\mathrm{ED}_{50}$ was $0.1 \mathrm{mg} /$ $\mathrm{kg}$ for Levo-BUP and $0.43 \mathrm{mg} / \mathrm{kg}$ for QX-OH. Compared with QX-314, the margin of safety for QX-OH broadened to some extent. However, the SI of QX-314 and QX-OH was significantly lower than that of Levo-BUP and LL-1. There were no significant differences between Levo-BUP and LL-1 for SI. That is, the dose needed to elicit cardiotoxicity by Levo-BUP or LL-1 was significantly higher than the dose resulting in sensory blockade (Table 1).

\section{Nonsynergistic effect was revealed between $\mathrm{QX}-\mathrm{OH}$ and Levo-BUP}

For $\mathrm{CD}_{50 \mathrm{C}}, \gamma$ between Levo-BUP and QX-OH was 1.537. With addition of Levo-BUP, the $\mathrm{CD}_{50 \mathrm{C}}$ of QX-OH was above the additive line, suggesting an antagonistic effect between Levo-BUP and QX-OH (Figure 2A). For $\mathrm{CD}_{50 \mathrm{~A}}$, the $\gamma$ between Levo-BUP and QX-OH was 1.202. With addition of LevoBUP, the $\mathrm{CD}_{50 \mathrm{~A}}$ of $\mathrm{QX}-\mathrm{OH}$ was above or on the additive line, indicating a nonsynergistic effect between Levo-BUP and QX-OH (Figure 2B).

\section{The ability to resuscitate rats}

Thirty-four rats were studied in experiment 3, but 2 rats in the QX-314 group were excluded because they did not develop asystole. Groups were similar in terms of weight, heart rates, systolic blood pressure, RPP, and acid-base status at baseline (Table 2).
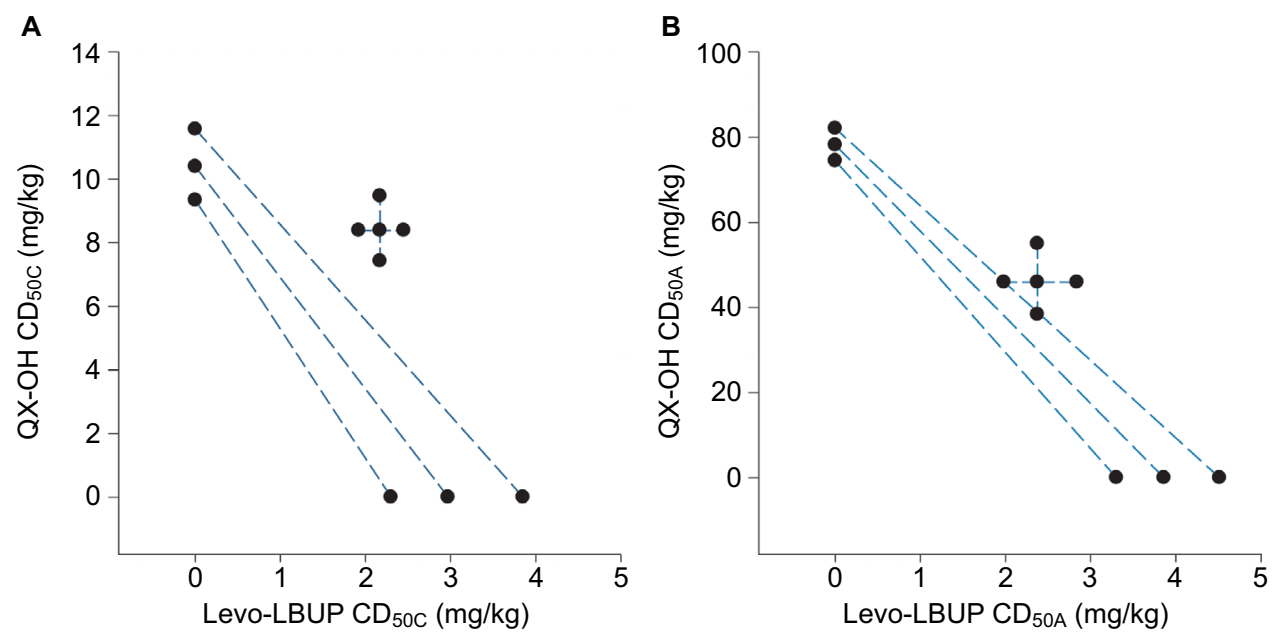

Figure 2 Interaction between Levo-BUP and QX-OH on cardiac effects in rat.

Notes: (A and B) Isobologram between Levo-BUP and QX-OH for cardiotoxicity and asystole, respectively. The $\mathrm{CD}_{50}$ of $\mathrm{QX}-\mathrm{OH}$ is on the vertical axis and the $\mathrm{CD}_{50}$ of Levo-BUP is on the horizontal axis. $\mathrm{CD}_{50 C}$, median dose resulting in appearance of cardiotoxicity; $\mathrm{CD}_{50 \mathrm{~A}}$, median dose resulting in asystole.

Abbreviation: Levo-BUP, levobupivacaine.

Table 2 Baseline values of major parameters for all 4 groups

\begin{tabular}{|c|c|c|c|c|c|c|c|c|}
\hline $\begin{array}{l}\text { Groups } \\
(N=8)\end{array}$ & Weight (g) & HR (beats/min) & SBP (mmHg) & $\begin{array}{l}\text { RPP }(\mathrm{mmHg} \\
\left.\text { beats }^{-1} \cdot \mathrm{min}^{-1}\right)\end{array}$ & PH & $\begin{array}{l}\mathrm{PaCO}_{2} \\
(\mathrm{mmHg})\end{array}$ & $\begin{array}{l}\mathrm{PaO}_{2} \\
(\mathrm{mmHg})\end{array}$ & $\begin{array}{l}\text { Lactate } \\
(\mathrm{mmol} / \mathrm{L})\end{array}$ \\
\hline Levo-BUP & $263 \pm 13$ & $390 \pm 12$ & $126 \pm 7$ & $48987 \pm 3342$ & $7.31 \pm 0.03$ & $31.41 \pm 1.15$ & $228.63 \pm 29.02$ & $1.46 \pm 0.48$ \\
\hline QX-3I4 & $263 \pm 5$ & $381 \pm 14$ & $124 \pm 8$ & $46178 \pm 2359$ & $7.30 \pm 0.01$ & $30.11 \pm 1.10$ & $212.00 \pm 26.74$ & $1.48 \pm 0.42$ \\
\hline $\mathrm{QX}-\mathrm{OH}$ & $264 \pm 8$ & $377 \pm 23$ & $|3| \pm 6$ & $49284 \pm 4159$ & $7.31 \pm 0.02$ & $30.54 \pm 2.81$ & $215.38 \pm 27.43$ & $1.44 \pm 0.42$ \\
\hline LL-I & $271 \pm 23$ & $381 \pm 21$ & $124 \pm 10$ & $47207 \pm 4104$ & $7.31 \pm 0.01$ & $30.55 \pm 1.20$ & $239.75 \pm 39.23$ & $1.05 \pm 0.47$ \\
\hline
\end{tabular}

Notes: All values are mean \pm SD. Baseline values for major parameters showed no significant differences among the 4 groups. LL-I, mixture of QX-OH and Levo-BUP; $\mathrm{PaCO}_{2}$, partial pressure of carbon dioxide in arterial blood; $\mathrm{PaO}_{2}$, partial pressure of oxygen in arterial blood; RPP, rate-pressure product $=\mathrm{SBP} \times \mathrm{HR}$.

Abbreviations: HR, heart rate; Levo-BUP, levobupivacaine; SBP, systolic blood pressure; SD, standard deviation. 
ROSC was attained initially by 8 of 8 rats in the LevoBUP group, 3 of 8 in the QX-314 group, 6 of 8 in the QX-OH group, and 8 of 8 in the LL-1 group (Table 3). There were no significant differences among Levo-BUP, QX-OH, and LL-1 groups or between QX-314 vs. QX-OH groups $(P>$ 0.05). Compared with the QX-314 group, the number of rats achieving successful resuscitation increased significantly in Levo-BUP and LL-1 groups $(P<0.05)$. Sustained recovery was achieved in the Levo-BUP group. However, no rats could maintain RPP $\geq 30 \%$ baseline in QX-314, QX-OH, and LL-1 groups at 10, 20, and $50 \mathrm{~min}$ after ROSC, respectively. Moreover, asystole occurred again in some rats that had attained spontaneous circulation: 2 of 3 in the QX-314 group, 4 of 6 in the QX-OH group, and 2 of 8 in the LL-1 group.

$\mathrm{T}_{\text {asystole }}$ of the $\mathrm{QX}-\mathrm{OH}$ group was significantly longer than that of other groups $(P<0.05)$ (Figure $3 \mathrm{~A}) . \mathrm{T}_{\text {recovery }}$ of the LL-1 group was significantly longer than that of QX-314 and QX-OH groups $(P<0.05)$. However, no significant differences were seen between Levo-BUP vs. other groups (Figure 3B).

The dose of epinephrine required for successful resuscitation was $38.75 \pm 11.26,23.33 \pm 5.77,28.33 \pm 11.69$, and $65.00 \pm 32.51 \mathrm{mg} / \mathrm{kg}$ in Levo-BUP, QX-314, QX-OH, and LL-1 groups, respectively (Figure 3C). Compared with QX-OH group, more epinephrine was needed to resuscitate rats in the LL-1 group $(P<0.05)$.

Figure 3D highlights the percent arrhythmia duration recorded after ROSC. Rats receiving Levo-BUP had no significant arrhythmia after ROSC. Percent arrhythmia duration was significantly less than in the Levo-BUP group than in the other groups $(P<0.05)$. However, there were no significant differences among QX-314, QX-OH, or LL-1 groups.

There were no significant differences in $\mathrm{pH}$, partial pressure of carbon dioxide, or partial pressure of oxygen among all groups $(P>0.05)$ except that $\mathrm{pH}$ in the LL-1 group and partial pressure of carbon dioxide in the QX-OH group at $60 \mathrm{~min}$ after ROSC were lower than those in the Levo-BUP group $(P<0.05)$. Lactate levels before asystole and after ROSC are shown in Table 4. Compared with the Levo-BUP group, lactate levels showed significant increases in the QX-OH group at 15 and 60 min and in the LL-1 group from 30 to 60 min after resuscitation $(P<0.05)$.

\section{Discussion}

Our study elicited 3 main findings. First, the order of safety was LL-1 » Levo-BUP > QX-OH > QX-314. Second, the interaction between QX-OH and Levo-BUP for cardiac effects was nonsynergistic. Third, the initial ability to resuscitate rats in which asystole had been induced by Levo-BUP, QX-OH, or LL-1 was similar. However, it was more difficult to resuscitate rats after induction of systole by QX-314 than by Levo-BUP or LL-1. Moreover, advanced life support might be needed after ROSC in QX-314-, QX-OH-, and LL-1-induced asystole.

$\mathrm{CD}_{50 \mathrm{C}}$ was measured by observing the ECG changes to identify the appearance of cardiotoxicity after administration of different local anesthetics. These changes occurred at a lower concentration range..$^{15}$ Cheung et al ${ }^{9}$ reported that QX-314 elicits cardiotoxicity in mice with a $\mathrm{CD}_{50}$ of $10.6 \mathrm{mg} /$ $\mathrm{kg}$, a value that is in accordance with our results. However, they observed cardiotoxicity only $30 \mathrm{~s}$ after injection of QX-314, which was considerably shorter than our observing time (the time to peak of QX-314 was $\sim 1-3 \mathrm{~min}$ ).

According to the $\mathrm{CD}_{50 \mathrm{C}}$ and $\mathrm{CD}_{50 \mathrm{~A}}$, the potency for cardiac effects was Levo-BUP $>$ QX-314 $\geq$ QX-OH (Table 1). The potency for cardiotoxicity of local anesthetics is closely associated with the lipid solubility of drugs. ${ }^{16}$ Compared with QX-314 and QX-OH, Levo-BUP is more lipid soluble, which could be why the cardiotoxicity of Levo-BUP was more severe than that of the other drugs we tested. The lipid solubility of QX-OH is less than that of QX-314, which reduces the cardiotoxicity of QX-OH.

It is not sufficient to evaluate safety based merely on the absolute dose required to elicit cardiotoxicity. The margin of safety also needs to be considered. In our study, even though the absolute dose of Levo-BUP resulting in cardiotoxicity was significantly lower than that of QX-314 or QX-OH (2.97 vs. 10.00 or 2.97 vs. $10.40 \mathrm{mg} / \mathrm{kg}$ ), the SI

Table 3 Animals maintaining spontaneous circulation after attaining initially successful resuscitation for each group and time

\begin{tabular}{|c|c|c|c|c|c|c|c|c|c|c|c|c|}
\hline \multirow[t]{2}{*}{ Groups $(\mathbf{N}=\mathbf{8})$} & \multicolumn{12}{|c|}{ After ROSC (min) } \\
\hline & 5 & 10 & 15 & 20 & 25 & 30 & 35 & 40 & 45 & 50 & 55 & 60 \\
\hline Levo-BUP & 8 & 8 & 8 & 8 & 8 & 8 & 8 & 8 & 8 & 8 & 8 & 8 \\
\hline$Q X-314$ & $3 *$ & $0 *$ & $0 *$ & $0 *$ & $0^{*}$ & $0 *$ & $0 *$ & $0 *$ & $0 *$ & $0 *$ & $0^{*}$ & 0* \\
\hline QX-OH & 6 & 4 & $I^{*}$ & $0 *$ & $0 *$ & $0 *$ & $0 *$ & $0 *$ & $0 *$ & $0 *$ & $0 *$ & 0* \\
\hline LL-I & $8^{2}$ & $7^{2}$ & $6^{\text {\&\# }}$ & $3^{*}$ & $3^{*}$ & $3^{*}$ & $2^{*}$ & $I^{*}$ & $I^{*}$ & $0 *$ & $0^{*}$ & $0 *$ \\
\hline
\end{tabular}

Notes: $* P<0.05$ vs. Levo-BUP; ${ }^{~} P<0.05$ vs. $Q X-314$; ${ }^{*} P<0.05$ vs. QX-OH. LL-I, mixture of $Q X-O H$ and Levo-BUP.

Abbreviations: Levo-BUP, levobupivacaine; ROSC, return of spontaneous circulation. 

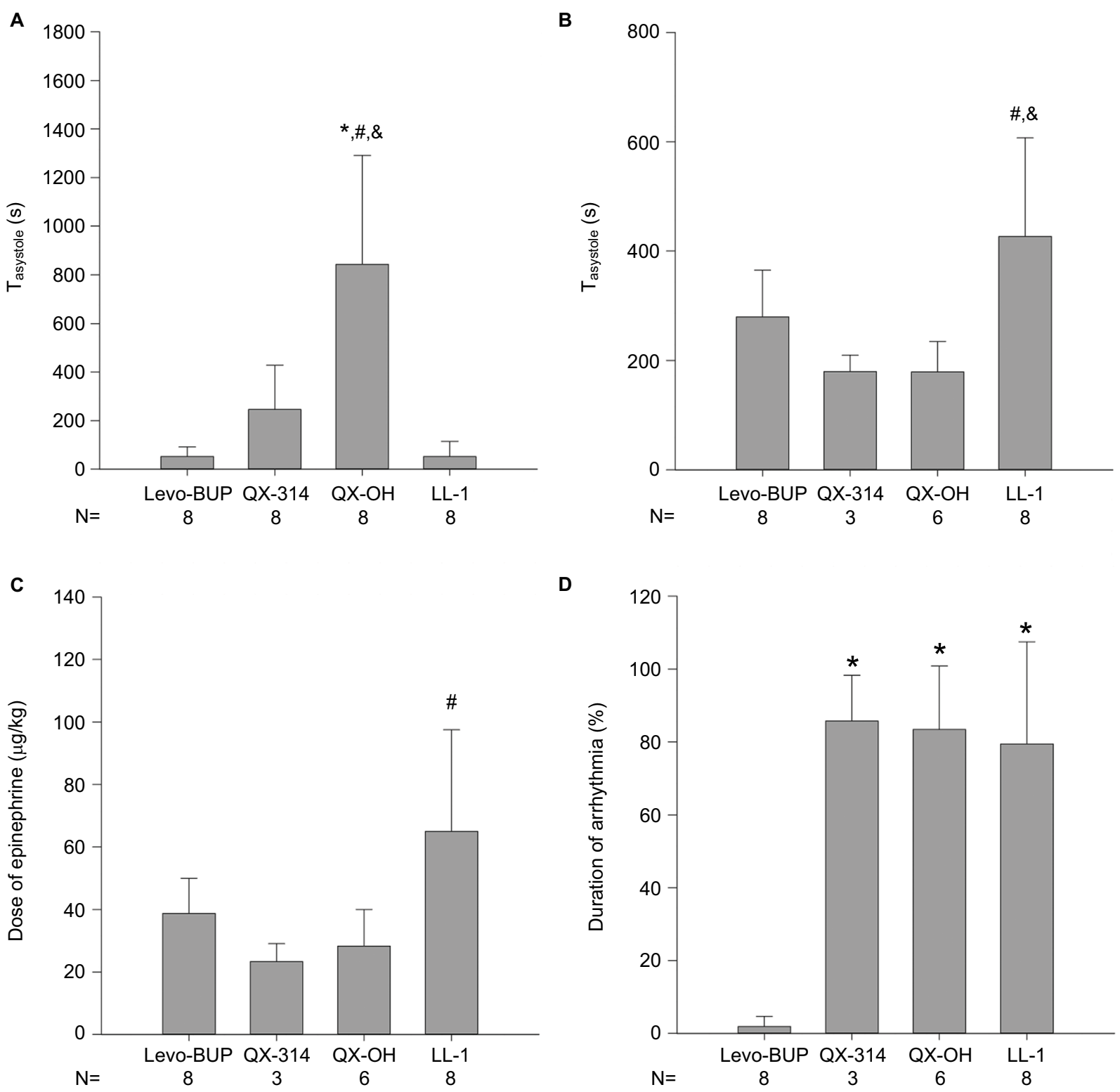

Figure 3 (A) $\mathrm{T}_{\text {arssole }}$ and (B) $\mathrm{T}_{\text {recovery }}$ dose of epinephrine required for (C) successful resuscitation and (D) duration of arrhythmia (\%) after ROSC are plotted. Notes: $* P<0.05$ vs. Levo-BUP; $\& P<0.05$ vs. QX-314; $\# P<0.05$ vs. $Q X-O H$. $T_{\text {asystele }}$, time from initiation of injection of local anesthetics to asystole; $T_{\text {recovery }}$, time from the start of asystole to ROSC; LL-I, mixture of QX-OH and Levo-BUP; N, number of animals.

Abbreviations: ROSC, return of spontaneous circulation; Levo-BUP, levobupivacaine.

Table 4 Lactate level before asystole and after ROSC (mmol/L)

\begin{tabular}{|c|c|c|c|c|c|}
\hline \multirow[t]{2}{*}{ Groups } & \multirow[t]{2}{*}{ Base } & \multicolumn{4}{|l|}{ After ROSC (min) } \\
\hline & & 15 & 30 & 45 & 60 \\
\hline Levo-BUP & $1.46 \pm 0.48(n=8)$ & $1.69 \pm 0.69(n=8)$ & $1.00 \pm 0.94(n=8)$ & $0.99 \pm 0.87(n=8)$ & $1.40 \pm 0.95(n=8)$ \\
\hline QX-314 & $1.48 \pm 0.42(n=8)$ & $3.80(n=1)$ & $6.80(n=1)$ & $6.90(n=1)$ & $7.60(n=1)$ \\
\hline $\mathrm{QX}-\mathrm{OH}$ & $1.44 \pm 0.42(n=8)$ & $4.23 \pm 1.85 *(n=4)$ & $3.50 \pm 1.21(n=2)$ & $3.35 \pm 1.77(n=2)$ & $5.90 \pm 1.84 *(n=2)$ \\
\hline LL-I & $1.05 \pm 0.47(n=8)$ & $2.30 \pm 1.18^{\#}(n=8)$ & $3.03 \pm|.7| *(n=8)$ & $3.47 \pm 2.40 *(n=7)$ & $3.92 \pm 2.53 *(n=6)$ \\
\hline
\end{tabular}

Notes: All values are mean \pm SD. The comparison between groups was omitted when the number of rats in any group was $<2$. $* P<0.05$ vs. Levo-BUP; $\# P<0.05$ vs. $Q X-$ $\mathrm{OH}$. LL-I, mixture of QX-OH and Levo-BUP.

Abbreviations: Levo-BUP, levobupivacaine; ROSC, return of spontaneous circulation; SD, standard deviation.

of Levo-BUP was significantly greater than that of QX-314 (10.60 vs. 1.20$)$ or QX-OH (10.60 vs. 1.44$)$. However, the SI of LL-1 was similar to that of Levo-BUP, suggesting that the safety of co-application of QX-OH and Levo-BUP was increased significantly compared with administration of QX-OH alone. 
Interestingly, nonsynergistic interaction was found between Levo-BUP and QX-OH with regard to cardiac effects. Systemic toxic effects of local anesthetic combinations appear to have additive effects. ${ }^{17}$ However, a mixture of BUP and LIDO can impair the intraventricular conduction parameters to a lesser extent than that for BUP itself. ${ }^{18}$ These different observations might have been resulted (at least in part) because of differences in experimental design and ethnicities of participants. From an electrophysiological viewpoint, local anesthetics elicit cardiotoxicity primarily by blocking sodium channels. ${ }^{19}$ LIDO blocks sodium channels in a "fast-in, fast-out" fashion, whereas BUP blocks these channels in a "slow-in, slow-out" manner in low concentrations or a "fast-in, slow-out" manner at high concentrations. ${ }^{20}$ Therefore, competitive blockade is present between BUP and LIDO. QX-OH, a derivative of LIDO, may compete with Levo-BUP for blockade of sodium channels. Meanwhile, the decreasing lipid solubility of QX-OH resulted in an extension of onset time on blocking the sodium channels of cardiac cells, which was longer than that of Levo-BUP. QX-OH might block sodium channels in a "more slow-in, more slow-out" fashion. Thus, the moment when QX-OH produced its peak effect was later than that of Levo-BUP or was even in the recovery stage of Levo-BUP-induced cardiotoxicity. These phenomena could explain, to a certain extent, the antagonism between QX-OH and Levo-BUP.

The interaction index $(\gamma)$ between QX-OH and LevoBUP for asystole was less than that for the appearance of cardiotoxicity (1.202 vs. 1.537), suggesting that the degree of antagonism decreased with increasing dose of QX-OH and Levo-BUP. Moreover, the $\mathrm{CD}_{50 \mathrm{~A}}$ of QX-OH was above or on the additivity line, indicating a nonsynergistic effect between Levo-BUP and QX-OH. However, the reason for this effect is not known. It has been reported that QX-314 alone at relatively high concentrations can activate TRP channels without any adjuvant drug and may penetrate slowly through lipid membranes. ${ }^{1,21}$ Thus, the time to peak effects of QX-OH was shortened due to activation of TRP channels induced by a relatively massive dose of QX-OH. Thus, the time to peak effects of QX-OH might overlap with that of Levo-BUP when LL-1 was administrated, inducing a nonsynergistic effect.

The nonsynergistic effect was of great importance for the safety of the drug co-application. Compared with QX-OH/Levo-BUP alone, the co-application of QX-OH and Levo-BUP produced the same or the lighter effects on cardiac functions under the same condition without affecting the potency of local anesthesia (according to $\mathrm{ED}_{50}$, the $\gamma$ between QX-OH and Levo-BUP for sensory blockade was 0.817 , indicating a synergistic effect on local anesthesia). We therefore supposed that the compound preparation was reasonable in terms of effectiveness and safety. However, the precise mechanism of this interaction affecting the cardiac conductivity or contractility was still unknown in this study.

With regard to the mechanism of death, asystole occurred rapidly in a fashion of disappearance of cardiac electrical activity after administration of Levo-BUP or LL-1, whereas QX-314 and QX-OH brought about ventricular fibrillation a few minutes after injection of these drugs. Ventricular fibrillation did not occur in the LL-1 group, suggesting that LevoBUP in LL-1 had a very important role in eliciting asystole. We found that the ranking of $\mathrm{T}_{\text {asystole }}$ was QX-OH $>$ QX-314 $>$ LL-1 » Levo-BUP. Local anesthetics need access to intracellular domains to block voltage-gated sodium channels. ${ }^{22}$ However, QX-314 cannot block sodium channels if applied extracellularly. ${ }^{4}$ This difference is due to the physicochemical properties of drugs at least in part, especially lipid solubility and charge. ${ }^{23} \mathrm{~T}_{\text {asystole }}$ in the LL-1 group was shorter than that in the QX-OH group, but it is not known whether this effect was due to the Levo-BUP in LL-1 or Levo-BUP-induced cellular entry of QX-OH or both.

Our results suggested that the ability to resuscitate initially rats was similar among Levo-BUP, LL-1, and QX-OH groups. QX-314-induced asystole was relatively more difficult to resuscitate. However, sustainable arrhythmia occurred in QX-314, QX-OH, and LL-1 groups, but not in the LevoBUP group, after ROSC, which led to circulatory collapse again. According to the manner of asystole or duration of arrhythmia (\%) after ROSC in QX-314, QX-OH, and LL-1 groups, arrhythmia appeared to be the primary mechanism of cardiotoxicity induced by QX-314 and QX-OH. Even though epinephrine can increase the number of ECG abnormalities after ROSC, ${ }^{24}$ we think that arrhythmogenicity is the internal characteristic of QX-314 and QX-OH because-ventricular fibrillation occurred before use of epinephrine.

Similarly, Groban et a ${ }^{25}$ reported that continued therapy was required in $86 \%$ of dogs receiving LIDO compared with only $10 \%-30 \%$ of dogs receiving BUP, Levo-BUP, or ropivacaine (ROP). They thought that "pump failure" was the cause of death in LIDO-treated dogs. However, we think that arrhythmia is the primary mechanism of cardiotoxicity induced by QX-314 and QX-OH. Local anesthetics decrease ventricular conduction in a stereospecific manner and decrease contractility in a non-stereospecific manner based on stereospecificity for blockade of sodium and potassium channels. ${ }^{26-28}$ Therefore, it is not known whether variation of 
the molecular structure of LIDO leads to the stereospecificity of the blockade of ion channels with LIDO.

In a word, arrhythmogenicity of QX-314 and QX-OH was the reason why sustainable circulation could not be maintained after ROSC in QX-314, QX-OH, and LL-1 groups. It tells us that anti-arrhythmia and other continued therapy may be important for the treatment of severe cardiotoxicity induced by QX-314, QX-OH, and LL-1. Also, it will be beneficial to monitor the dynamic change of vital signs for a relatively longer time after ROSC. Even though circulatory collapse occurred again in the LL-1 group due to the arrhythmogenicity of QX-OH, it cannot deny absolutely the use of LL-1 because the dose required to elicit asystole was much larger than the dose produced local anesthesia.

Our study had 3 main limitations. First, isoflurane was used for basal anesthesia. We cannot rule out completely the effects of isoflurane on experimental results. However, we maintained a relatively low concentration and identical conditions between groups. Second, we did not monitor blood pressure of rats in measuring $\mathrm{CD}_{50 \mathrm{C}}$ and $\mathrm{CD}_{50 \mathrm{~A}}$. As we know, ECG changes induced by local anesthetics occurred in a low concentration range. Asystole occurred in a fashion of the disappearance of cardiac electrical activity or ventricular fibrillation in a preliminary experiment, which was confirmed by ECG. Third, we did not use lipid emulsions in our resuscitation sequence. Even though lipid emulsions have been approved by many anesthetists, epinephrine remains the first-line drug for cardiopulmonary resuscitation because of its positive chronotropy, inotropy, and vasopressor effects. However, epinephrine over a threshold dose near $10 \mu \mathrm{g} / \mathrm{kg}$ impairs lipid resuscitation from BUP overdose. ${ }^{29}$ Moreover, the "lipid sink" theory is the mechanism of lipid on the reversal of local anesthetic cardiotoxicity. ${ }^{30}$ Some scholars have found no beneficial effects of lipid infusion on the less lipophilic mepivacaine- or ROP-induced asystole, concluding that lipophilicity of local anesthetics has a marked impact on the efficacy of lipid infusion to treat asystole induced by these drugs. ${ }^{10,31} \mathrm{QX}-\mathrm{OH}$ is a less lipophilic drug. So, we did not choose lipid emulsions in our study.

\section{Conclusion}

The SI of LL-1 was similar to that of Levo-BUP. The cardiac effects of QX-OH and Levo-BUP were nonsynergistic. Initial successful resuscitation could be achieved in QX-OH- and LL-1-induced asystole. Though circulatory collapse occurred again, that did not deny the use of QX-OH or LL-1 because the dose required to elicit asystole was much larger than the dose used to elicit local anesthesia. Furthermore, results suggested that advanced life support might be needed after ROSC. Further studies are needed to define what kind of advanced life support is needed after ROSC to maintain stable circular function and the precise mechanism of cardiotoxicity with respect to cellular/molecular actions and pharmacokinetics.

\section{Acknowledgments}

We sincerely thank the members of the new drug research team for their contributions to this study. This article was financially supported by National Science and Technology Major Project, Ministry of Science and Technology of the People's Republic of China (number 2014ZX09101-001), Beijing, People's Republic of China.

\section{Author contributions}

All authors contributed toward data analysis, drafting and revising the paper and agree to be accountable for all aspects of the work.

\section{Disclosure}

The authors report no conflicts of interest in this work.

\section{References}

1. Lim T, Macleod B, Ries C, Schwarz S. The quaternary lidocaine derivative, QX-314, produces long-lasting local anesthesia in animal models in vivo. Anesthesiology. 2007;107(2):305-311.

2. Zhao Y, Zhou C, Liu J, et al. The quaternary lidocaine derivative QX-314 produces long-lasting intravenous regional anesthesia in rats. PLoS One. 2014;9(6):e99704.

3. Nakagawa H, Hiura A. Comparison of the transport of QX-314 through TRPA1, TRPM8, and TRPV1 channels. J Pain Res. 2013;6: 223-230.

4. Ries C, Pillai R, Chung C, Wang J, MacLeod B, Schwarz S. QX-314 produces long-lasting local anesthesia modulated by transient receptor potential vanilloid receptors in mice. Anesthesiology. 2009;111(1): $122-126$.

5. Welk E, Petsche U, Fleischer E, Handwerker H. Altered excitability of afferent C-fibres of the rat distal to a nerve site exposed to capsaicin. Neurosci Lett. 1983;38(3):245-250.

6. Brenneis C, Kistner K, Puopolo M, et al. Bupivacaine-induced cellular entry of QX-314 and its contribution to differential nerve block. $B r J$ Pharmacol. 2014;171(2):438-451.

7. Vijay BS, Mitra S, Jamil SN. Refractory cardiac arrest due to inadvertent intravenous injection of $0.25 \%$ bupivacaine used for local infiltration anesthesia. Anesth Essays Res. 2013;7(1):130-132.

8. Bruce R. An up-and-down procedure for acute toxicity testing. Fundam Appl Toxicol. 1985;5(1):151-157.

9. Cheung HM, Lee SM, MacLeod BA, Ries CR, Schwarz SK. A comparison of the systemic toxicity of lidocaine versus its quaternary derivative QX-314 in mice. Can J Anesth. 2011;58(5):443-450.

10. Aumeier C, Kasdorf B, Gruber M, et al. Lipid emulsion pretreatment has different effects on mepivacaine and bupivacaine cardiac toxicity in an isolated rat heart model. Br J Anaesth. 2013;112(4):735-741.

11. Ohmura S, Kawada M, Ohta T, Yamamoto K, Kobayashi T. Systemic toxicity and resuscitation in bupivacaine-, levobupivacaine-, or ropivacaine-infused rats. Anesth Analg. 2001;93(3):743-748. 
12. Lichtman AH. The up-and-down method substantially reduces the number of animals required to determine antinociceptive ED 50 values. J Pharmacol Toxicol Methods. 1998;40(2):81-85.

13. Gessner PK. Isobolographic analysis of interactions: an update on applications and utility. Toxicology. 1995;105(2):161-179.

14. Tallarida RJ. The interaction index: a measure of drug synergism. Pain. 2002;98(1):163-168.

15. Covino BG. Ultralong-acting local anesthetic agents. J Am Soc Anesthesiol. 1981;54(4):263-264.

16. Tsuchiya H, Ueno T, Mizogami M, Takakura K. Local anesthetics structure-dependently interact with anionic phospholipid membranes to modify the fluidity. Chem Biol Interact. 2010;183(1):19-24.

17. Mets B, Janicki PK, James MF, Erskine R, Sasman B. Lidocaine and bupivacaine cardiorespiratory toxicity is additive: a study in rats. Anesth Analg. 1992;75(4):611-614.

18. Krikava I, Jarkovský J, Stourac P, Nováková M, Sevcik P. The effects of lidocaine on bupivacaine-induced cardiotoxicity in the isolated rat heart. Physiol Res. 2010;59(suppl 1):S65-S69.

19. Fukuda K, Nakajima T, Viswanathan PC, Balser JR. Compound-specific $\mathrm{Na}+$ channel pore conformational changes induced by local anaesthetics. J Physiol. 2005;564(1):21-31.

20. Clarkson CW, Hondeghem LM. Mechanism for bupivacaine depression of cardiac conduction: fast block of sodium channels during the action potential with slow recovery from block during diastole. Anesthesiology. 1985;62(4):396-405.

21. Rivera-Acevedo RE, Pless SA, Ahern CA, Schwarz SK. The quaternary lidocaine derivative, QX-314, exerts biphasic effects on transient receptor potential vanilloid subtype 1 channels in vitro. JAm Soc Anesthesiol. 2011;114(6):1425-1434.
22. Butterworth JT, Strichartz GR. Molecular mechanisms of local anesthesia: a review. Anesthesiology. 1990;72(4):711-734.

23. Hille B. Local anesthetics: hydrophilic and hydrophobic pathways for the drug-receptor reaction. J Gen Physiol. 1977;69(4):497-515.

24. de Queiroz Siqueira M, Chassard D, Musard H, et al. Resuscitation with lipid, epinephrine, or both in levobupivacaine-induced cardiac toxicity in newborn piglets. Br J Anaesth. 2014;112(4):729-734.

25. Groban L, Deal DD, Vernon JC, James RL, Butterworth J. Cardiac resuscitation after incremental overdosage with lidocaine, bupivacaine, levobupivacaine, and ropivacaine in anesthetized dogs. Anesth Analg. 2001;92(1):37-43.

26. Kariya N, Cosson C, Mazoit J-X. Comparative effect of lidocaine, bupivacaine and RAC 109 on myocardial conduction and contractility in the rabbit. Eur J Pharmacol. 2012;691(1):110-117.

27. Valenzuela C, Delpon E, Tamkun MM, Tamargo J, Snyders DJ. Stereoselective block of a human cardiac potassium channel (Kv1. 5) by bupivacaine enantiomers. Biophys J. 1995;69(2):418.

28. Valenzuela C, Snyders DJ, Bennett PB, Tamargo J, Hondeghem LM. Stereoselective block of cardiac sodium channels by bupivacaine in guinea pig ventricular myocytes. Circulation. 1995;92(10):3014-3024.

29. Hiller DB, Di Gregorio G, Ripper R, et al. Epinephrine impairs lipid resuscitation from bupivacaine overdose: a threshold effect. Anesthesiology. 2009;111(3):498-505.

30. Weinberg G, Lin B, Zheng S, et al. Partitioning effect in lipid resuscitation: further evidence for the lipid sink. Crit Care Med. 2010;38(11): 2268-2269.

31. Zausig YA, Zink W, Keil M, et al. Lipid emulsion improves recovery from bupivacaine-induced cardiac arrest, but not from ropivacaine-or mepivacaine-induced cardiac arrest. Anesth Analg. 2009;109(4):1323-1326.
Journal of Pain Research

\section{Publish your work in this journal}

The Journal of Pain Research is an international, peer reviewed, open access, online journal that welcomes laboratory and clinical findings in the fields of pain research and the prevention and management of pain. Original research, reviews, symposium reports, hypothesis formation and commentaries are all considered for publication.

\section{Dovepress}

The manuscript management system is completely online and includes a very quick and fair peer-review system, which is all easy to use. Visit http://www.dovepress.com/testimonials.php to read real quotes from published authors. 